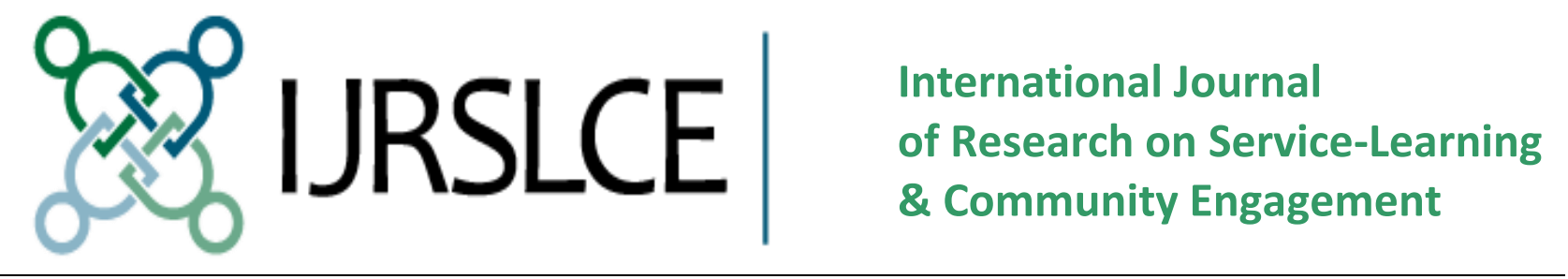

Volume 8, Issue 1 | 2020 | ISSN: 2374-9466 | https://ijrslce.scholasticahq.com/

The International Journal of Research on Service-Learning and Community Engagement (IJRSLCE) is a publication of the International Association for Research on Service-Learning and Community Engagement (IARSLCE). Currently published annually, IJRSLCE is dedicated to disseminating high-quality research focused on service-learning; campus-community engagement; and the promotion of active, effective citizenship through education.

IARSLCE promotes high-quality transdisciplinary research that reflects a wide range of approaches and forms - research that builds the capacity of scholars, practitioners, and community partners to engage in such research.

\title{
EDITORIAL TEAM
}

\section{EDITORS}

Glenn A. Bowen

Clayton Hurd

\section{SECTION EDITORS}

Burton A. Bargerstock

Henry R. Cunningham

Susan C. Harris

J.R. Jamison

Carol Hok Ka Ma

Jessica Mann

Paul H. Matthews

Phillip Motley

Megan Jeanette Myers

Billy Osteen

Heidi Whitford
Barry University (Florida, USA)

Campus Compact (Massachusetts, USA)

Michigan State University (Michigan, USA)

University of Louisville (Kentucky, USA)

University of Southern California (California, USA)

Indiana Campus Compact (Indiana, USA)

Singapore University of Social Sciences (Singapore)

Duquesne University (Pennsylvania, USA)

University of Georgia (Georgia, USA)

Elon University (North Carolina, USA)

Iowa State University (Iowa, USA)

University of Canterbury (New Zealand)

Barry University (Florida, USA)

\section{EDITORIAL ASSISTANT}

Milad Mohebali

University of Iowa (Iowa, USA) 


\section{REVIEWERS}

Abderrahim Agnaou

Eileen Alexander

Lottie Baker

Becca Berkey

Alan Bloomgarden

Robert G. Bringle

Renee C. Brown

Kathryn Clements

Christiane Connors

Stephanie Budhai

Stéphane Cardinal

Thomas A. Dahan

Brian Davenport

Thomas DePaola

Tyler Derreth

Keristiena Dodge

Jeremy Doughty

Rebecca Dumlao

Antonio Estudillo

Bob Frigo

Norma Gaines-Hanks

Nickesia Gordon

Patrick Green

Kai Greene

Daniel Hall

Muawya Hussein

Angela James

Ethan Kolek

Morgan Lewing

Charles Liu

Lorraine McIlrath

Miles McNall

Milad Mohebali

Keith Morton

Drew Pearl

David Proctor

Dan Sarofian-Butin

Colton Strawser

Amanda Sturgill

Margaret-Mary Suentic Dowell

Serena Xiong
Al Akhawayn University in Ifrane (Morocco)

Xavier University (Ohio, USA)

The George Washington University (Washington, D.C., USA)

Northeastern University (Massachusetts, USA)

Elms College (Massachusetts, USA)

IUPUI (Indiana, USA)

Michigan State University (Michigan, USA)

Michigan State University (Michigan, USA)

Edmund Burke School (Washington, D.C., USA)

Neumann University (Pennsylvania, USA)

University of Ottawa (Ontario, Canada)

Rutgers University-Camden (New Jersey, USA)

Eastern Washington University (Washington, USA)

University of Southern California (California, USA)

Johns Hopkins University (Maryland, USA)

University of Nebraska at Omaha (Nebraska, USA)

University Studies Abroad Consortium (England, UK)

East Carolina University (North Carolina, USA)

Monmouth University (New Jersey, USA)

Elon University (North Carolina, USA)

University of Delaware (Delaware, USA)

Rochester Institute of Technology (New York, USA)

Loyola University Chicago (Illinois, USA)

California State University-Dominguez Hills (California, USA)

High Point University (North Carolina, USA)

Dhofar University (Oman)

University of Kwazulu-Natal (South Africa)

Florida International University (Florida, USA)

Texas A\&M University-Central Texas (Texas, USA)

Michigan State University (Michigan, USA)

NUI Galway (Ireland)

Michigan State University (Michigan, USA)

University of Iowa (Iowa, USA)

Providence College (Rhode Island, USA)

University of Alabama (Alabama, USA)

Kansas State University (Kansas, USA)

Merrimack College (Massachusetts, USA)

University of Texas at Arlington (Arlington, USA)

Elon University (North Carolina, USA)

Louisiana State University (Louisiana, USA)

University of Minnesota-Twin Cities (Minnesota, USA) 